\title{
Silencing of the polyamine catabolic key enzyme SSAT prevents CDK inhibitor-induced apoptosis in Caco-2 colon cancer cells
}

\author{
A. ÇOKER, E.D.ARISAN and N. PALAVAN-ÜNSAL \\ Department of Molecular Biology and Genetics, Science and Literature Faculty, \\ Istanbul Kultur University, Atakoy Campus, 34156 Istanbul, Turkey
}

Received October 18, 2011; Accepted January 18, 2012

DOI: $10.3892 / \mathrm{mmr} .2012 .768$

\begin{abstract}
Roscovitine and purvalanol are purine derivative cyclin-dependent kinase (CDK) inhibitors that induce apoptosis in various types of cancer cells. However, their impact on the apoptotic cell death mechanism requires further elucidation. Natural polyamines putrescine, spermidine and spermine play essential roles in the regulation of cell growth and proliferation. Increased levels of polyamines in cells are considered to be involved in cancer progression. Intracellular polyamine levels are under the control of several catabolic enzymes, such as spermidine/spermine-N-acetyl transferase (SSAT), acetylpolyamine oxidase (APAO) and spermine oxidase (SMO), which could be altered by several therapeutic drugs. However, the possible role of polyamines in drug-induced apoptosis has yet to be clarified. In the present study, our aim was to determine the modulation of the polyamine catabolic pathway related to CDK inhibitor-induced apoptosis in Caco- 2 cells. We found that roscovitine and purvalanol (each $20 \mu \mathrm{M}$ ) induced apoptosis by activating caspase- 9 and -3 , and inhibiting the mitochondrial membrane potential in Caco- 2 cells. CDK inhibitors decreased the intracellular putrescine and spermine levels without affecting spermidine levels. Although both roscovitine and purvalanol induced SSAT expression, they did not exert a significant effect on the APAO expression profile. SSAT transient silencing prevented roscovitine-induced apoptosis compared to parental cells. Thus, we concluded that roscovitine and purvalanol significantly induce apoptosis in Caco-2 cells by modulating the polyamine catabolism, and that SSAT could be an important target in evaluating the potential role of polyamines in apoptotic cell death.
\end{abstract}

Correspondence to: Dr Narcin Palavan-Ünsal, Department of Molecular Biology and Genetics, Science and Literature Faculty, Istanbul Kultur University, Atakoy Campus, 34156 Istanbul, Turkey E-mail: n.palavanunsal@iku.edu.tr

Key words: roscovitine, purvalanol, cyclin-dependent kinase inhibitors, polyamines, spermidine/spermine- $\mathrm{N}$-acetyl transferase, colon carcinoma

\section{Introduction}

Cyclin-dependent kinases (CDKs) play a pivotal role in the control of aberrant cell cycle and/or the proliferation mechanism in malignant cells (1). Aberrations in the function of CDKs have also been found in Alzheimer's and Parkinson's diseases, illnesses caused by viral infections, ischemia or other proliferative disorders (2-4). Therefore, small-molecule inhibitors have been designed to explore the molecular functions of CDKs within a thereapeutic framework.

Roscovitine (2-(1-ethyl-2-hydroxyethylamino)-6-benzylamino-9-isopro-pylpurine) is a new generation reversible inhibitor of CDKs, such as Cdc2, Cdk2, Cdk5 and Cdk7, by behaving as a competitor for ATP binding (5-8). Roscovitine triggers cell cycle arrest at the G1 and G2/M phases (6) and leads to apoptotic induction which can be determined in each phase of the cell cycle (9). It has also been shown that following roscovitine treatment, DNA synthesis is inhibited and induces apoptosis by causing nucleolar fragmentation in various cancer cells. Due to these cell growth-inhibiting activities, roscovitine is being considered as a potential anticancer agent and a suitable candidate for combination treatment models (10-13). In vitro studies have revealed that roscovitine is a promising therapeutic agent by inducing apoptosis in prostate cancer (14), breast cancer $(10,15)$ and leukemia cells (16). Roscovitine has also been presented as a sensitizing drug in combination with other conventional therapeutic options in the treatment of cancer $(11,13)$.

Purvalanol was recently designed as a protein kinase inhibitor with high selectivity for Cdc2 and CDK2 through competitive inhibition of ATP binding resulting in G2/M cell cycle arrest (17). Recent studies have shown that purvalanol may increase drug-induced apoptosis by inhibiting formation of CDK2/cyclin B and CDK2/cyclin A, and CDK2/cyclin E and CDK5/p35 complexes (18-20). Purvalanol treatment also led to significant downregulation of anti-apoptotic molecules, such as survivin, $\mathrm{Bcl}-\mathrm{x}_{\mathrm{L}}$ and $\mathrm{Bcl}-2$ by globally inhibiting RNA synthesis $(21,22)$. Purvalanol also induced the activation of caspase-dependent apoptosis by altering mitochondrial membrane functions in various cancer cell lines $(23,24)$.

The natural polyamines (PAs) putrescine (Put), spermidine $(\mathrm{Spd})$ and spermine $(\mathrm{Spm})$ are ubiquitous polycationic amine derivatives found in all eukaryotic cells $(25,26)$. PAs are considered essential elements for cell proliferation, differentiation 
and growth in normal and cancer cells (27-29). Their cellular levels are referred to as critical regulators of cell cycle, survival and death mechanisms (30). PA metabolic regulation is characterized by several enzyme activities. Ornithine decarboxylase (ODC) is a rate limiting enzyme which induces synthesis of Put from L-arginine (31). High accumulation of PAs in cells are regulated by PA catabolic pathway players, spermidine/spermine N1-acetyltransferase (SSAT), spermine oxidase (SMO) and polyamine oxidase (PAO). These enzymes induce excretion of acetylated PA derivatives or provide a back-conversion pathway by oxidizing several compounds in the cells (32). Previous reports have shown that PA depletion by specific inhibitor DL- $\alpha$-difluoromethylornithine (DFMO) treatment may increase the apoptotic efficiency of drugs (33). Therefore, PA metabolic pathway-targeted therapies are gaining importance in the increase of combination therapy efficiency in clinics $(34,35)$. However, the molecular mechanism involved in drug-induced apoptosis related to PA biosynthetic regulation has yet to be fully understood.

In the present study, we aimed to determine the potential role of CDK inhibitors, roscovitine and purvalanol, on the apoptotic cell death mechanism related to the PA catabolic pathway in Caco-2 colon carcinoma cells.

\section{Materials and methods}

Chemicals, antibodies and primers. Roscovitine (Sigma, St. Louis, MO, USA) and purvalanol (Tocris Bioscience, Bristol, UK) were dissolved in DMSO to make a 10-mM stock solution and stored at $-20^{\circ} \mathrm{C}$. Put, Spd and Spm standards were purchased from Sigma. 3,3-Dihexyloxacarbocyanine iodide ( $\mathrm{DiOC}_{6}$ ) was purchased from Calbiochem (La Jolla, CA, USA). Caspase inhibitors (each 10-mM stock solution), z-DEVDFMK (caspase-3), z-LEHD-FMK (caspase-9), z-VAD-FMK (general caspase) and Z-FA-FMK (negative caspase) were purchased from BD Biosciences (San Jose, CA, USA).

$\beta$-actin $(1: 2,000), \mathrm{Bcl}_{\mathrm{L}}(1: 1,000)$, Bax $(1: 1,000)$, PUMA $(1: 1,000)$, Bim $(1: 1,000)$, PARP $(1: 1,000)$, cleaved PARP $(1: 1,000)$, cleaved caspase-3 $(1: 1,000)$ and pro-caspase-3 $(1: 1,000)$ anti-rabbit antibodies were purchased from Cell Signaling Technology (CST; Danvers, MA, USA). ODC, SSAT and PAO anti-rabbit antibodies $(1: 2,000)$ were purchased from Santa Cruz Biotechnology (Santa Cruz, CA, USA). Horseradish peroxidase (HRP)-conjugated secondary antirabbit and anti-mouse antibodies $(1: 5,000)$ were from CST.

Cell culture. Caco-2 colon carcinoma cells (HTB-37) (ATCC) were maintained in minimal essential medium (PAN Biotech, Aidenbach, Germany) with $2 \mathrm{mM}$ L-glutamine, $20 \%$ fetal calf serum (PAN Biotech), $1 \%$ non-essential amino acids (Biological Industries) and 100 units/100 mg/ml penicillin/ streptomycin (Biological Industries, Kibbutz Beit-Haemek, Israel) and grown in the presence of $5 \% \mathrm{CO}_{2}$ in humidified air at $37^{\circ} \mathrm{C}$.

Cell viability assay. Cells were seeded in 96-well plates and co-treated with various concentrations of roscovitine or purvalanol $(0-50 \mu \mathrm{M})$ for $24 \mathrm{~h}$. The cytotoxic effect of CDK inhibitors on Caco-2 cells was determined by colorimetric 3-(4,5-dimethylthiazol-2-yl)-2,5-diphenyl-tetrazolium bromide (MTT; Roche, Indianapolis, IN, USA) cell proliferation assay, which is based on the conversion of MTT to MTT-formazan by mitochondrial enzymes. Absorbance was determined with a microplate reader at $570 \mathrm{~nm}$ (Bio-Rad, Hercules, CA, USA).

Apoptosis determination. Caco-2 cells $\left(1 \times 10^{4}\right)$ were seeded in 96-well plates, co-treated with $0-50 \mu \mathrm{M}$ roscovitine and purvalanol for $24 \mathrm{~h}$. Cytoplasmic histone-associated-DNA fragments (mono- and oligonucleosomes) were determined using Cell Death Detection ELISAPlus assay, according to the manufacturer's instructions (Roche). Briefly, cell lysates were placed in a streptavidin-coated microplate. A mixture of anti-histone-biotin and anti-DNA-POD was added and incubated for $2 \mathrm{~h}$ at $15-25^{\circ} \mathrm{C}$. Following the removal of unbound antibodies by a washing procedure, POD was determined photometrically at $405 \mathrm{~nm}$ with ABTS as substrate. In order to determine the DNA fragments following drug treatment in colon carcinoma cells, total DNA content was isolated.

Mitochondrial membrane potential assay. Caco-2 cells $\left(1 \times 10^{5}\right)$ were seeded in 12-well plates, allowed to attach overnight and treated with desired concentrations of roscovitine for $24 \mathrm{~h}$. Cells were washed once with $1 \mathrm{X}$ PBS and then stained with $\mathrm{DiOC}_{6}$ (Calbiochem) fluorescent probe. Mitochondrial membrane potential (MMP) loss was measured by Fluoroskan Ascent fluorometer (Thermo Labsystems, Beverly, MA, USA) (excitation/emission $=488 \mathrm{~nm} / 525 \mathrm{~nm})$.

Polyamine determination. PA content of the cells was determined by the benzoylation procedure using HPLC. Cells $\left(0.6 \times 10^{6}\right)$ were seeded in 6 -well plates and allowed to attach overnight. Caco- 2 cells were treated with roscovitine or purvalanol for $24 \mathrm{~h}$ and were then washed with $1 \mathrm{X}$ PBS. The scraped cell lysates were transferred into a new microfuge tube. Trichloroacetic acid (TCA; 50\%) was added to each sample (1:10, v:v). All samples were kept at $-20^{\circ} \mathrm{C}$ until the benzoylation process. Following benzoylation, samples were immediately run on HPLC using UV detector on $226 \mathrm{mV}$. The data obtained were evaluated according to internal standards 1,6-diaminoheptane and the standard curves of Put, Spd and Spm.

Preparation of siRNA transfection. Caco-2 colon carcinoma cells were placed in a 6 -well plate $24 \mathrm{~h}$ prior to transfection. Cells were transfected with different SSAT siRNA complexes that target SSAT-encoding genes SAT1 or SAT2 at two different mRNA binding sites (each 20 nM; Gene Globe, Qiagen, Heidelberg, Germany). The transfection ratio was 1:6 and transfection was performed (RNAifect; Qiagen) according to the manufacturer's protocol. After $48 \mathrm{~h}$ of incubation, the silencing effect was analyzed with western blot analysis.

Immunoblot analysis. Caco-2 colon carcinoma cells were treated with the appropriate concentrations of drugs. The samples were initially washed with ice-cold $1 \mathrm{X}$ PBS and lysed on ice in a solution containing $20 \mathrm{mM}$ Tris- $\mathrm{HCl}$ (pH 7.5), $150 \mathrm{mM} \mathrm{NaCl}$, Nonidet P-40 0.5\% (v/v), $1 \mathrm{mM}$ EDTA, $0.5 \mathrm{mM}$ PMSF, $1 \mathrm{mM}$ DTT, protease inhibitor cocktail (Complete; Roche). Following cell lysis, cell debris 
was removed by centrifugation for $15 \mathrm{~min}$ at $13,200 \mathrm{x} \mathrm{g}$, and protein concentrations were determined with a Bradford protein assay. Total protein lysates $(30 \mu \mathrm{g})$ were separated on a $12 \%$ SDS-PAGE and transferred onto PVDF membranes (Amersham Pharmacia Biotech, Little Chalfont, UK). The membranes were then blocked with $5 \%$ milk blocking solution in Tris buffer saline (TBS) Tween-20 (Sigma) and incubated with appropriate primary and HRP-conjugated secondary antibodies (CST) in antibody buffer containing 5\% (v/v) milk blocking solution. After washing with TBS Tween-20, proteins were analyzed using an enhanced chemiluminescence detection system (ECL or ECL-Advance; Amersham Pharmacia Biotech) and exposed to Hyperfilm-ECL (Amersham Pharmacia Biotech).

Statistical analysis. All samples were evaluated statistically using an Excel calculation file. The MTT and cell death ELISA assay results are shown as the means \pm standard deviation, and the Student's t-test was applied to evaluate the probability efficiency. Differences were regarded as statistically significant at $\mathrm{p}<0.05$.

\section{Results}

Roscovitine and purvalanol are potent apoptotic inducers. We used Caco-2 cells to investigate whether roscovitine or purvalanol decreases cell viability through the induction of apoptosis. To assess the cytotoxic effects of both CDK inhibitors, Caco- 2 cells were treated with roscovitine or purvalanol at various concentrations $(0-50 \mu \mathrm{M})$ for $24 \mathrm{~h}$ and MTT cell viability assay was assessed. CDK inhibitors decreased cell viability in a dose-dependent manner. According to the MTT, roscovitine and purvalanol (each $20 \mu \mathrm{M}$ ) decreased cell viability by $\sim 30 \%$ in Caco- 2 cells (Fig. 1A). To confirm that the decrease in cell viability was indeed due to apoptosis, we performed the Cell Death ELISA Plus assay (Fig. 1B). Roscovitine or purvalanol treatment led to a significant 2-fold increase in apoptotic cell death in Caco-2 cells compared to the untreated samples.

In order to understand CDK inhibitor-induced apoptosis due to activation of caspases, cells were co-treated with several caspase inhibitors (each $2.5 \mu \mathrm{M}$ ) and purvalanol or roscovitine for $24 \mathrm{~h}$; the MTT cell viability assay was then performed. Co-treatment with caspase inhibitors, z-VAD-FMK (pan-caspase inhibitor), z-LEHD-FMK (caspase-9 inhibitor), z-DEVD-FMK (caspase-3 inhibitor) significantly prevented roscovitine- or purvalanol-induced apoptosis, respectively (Fig. 2A). These observations were also confirmed by the determination of MMP loss by DiOC $_{6}$ staining. As shown in Fig. 2B, when the cells were treated with roscovitine $(20 \mu \mathrm{M})$ in the presence of each caspase inhibitor for $24 \mathrm{~h}$, roscovitine-induced MMP loss was prevented. To assess whether drug-induced apoptosis is mediated by caspases, the proteolytic activation of caspase- 3 was also examined in Caco-2 cells. Both CDK inhibitors induced cleavage of pro-caspase-3 to the active form (p19/17) (Fig. 2C). Involvement of apoptosis was further confirmed by the detection of PARP degradation in Caco-2 cells compared to untreated samples. Furthermore, CDK inhibitors were able to alter Bcl-2 family members (Fig. 2D) which are critical in
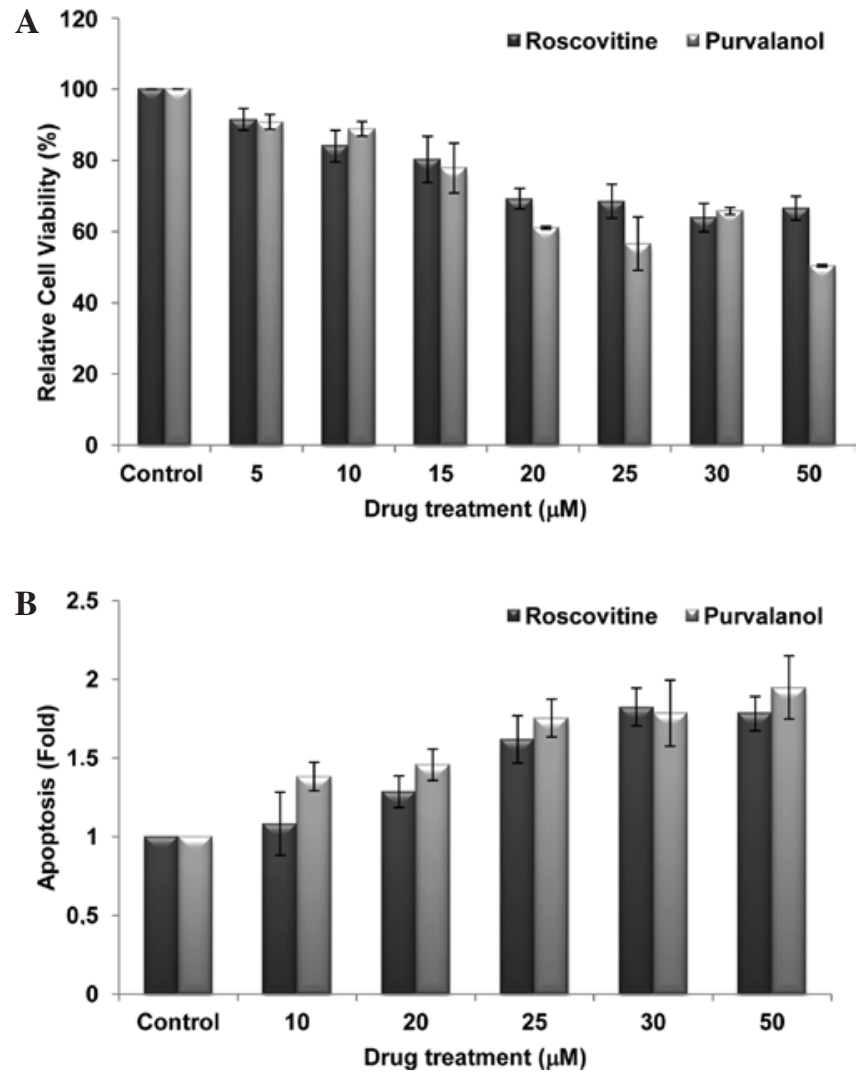

Figure 1. Roscovitine and purvalanol decrease cell viability and induce apoptosis in Caco-2 cells. (A) Cells were treated with roscovitine and purvalanol (each 0-50 $\mu \mathrm{M}$ ) for $24 \mathrm{~h}$. Columns represent the means \pm SD of two independent trials with at least five replicates. (B) Apoptotic cell death of Caco-2 cells was determined using the Cell Death Detection ELISA Plus assay following exposure of cells with CDK inhibitors (0-50 $\mu \mathrm{M})$. Columns represent the means $\pm \mathrm{SD}$ of two independent trials with at least two replicates.

the apoptotic decision in the cells. Although roscovitine did not alter Bcl- $\mathrm{X}_{\mathrm{L}}$ and Puma expression profiles, the exposure of cells to roscovitine led to upregulation of Bax expression in Caco-2 cells. Purvalanol upregulated both anti-apoptotic $\mathrm{Bcl}-\mathrm{X}_{\mathrm{L}}$ and pro-apopotic Bax and Puma protein expression profiles.

CDK inhibitors modulate polyamine metabolism in the drug-induced apoptotic mechanism. Since PAs are critical in cellular homeostasis and in conducting several signalling networks, including the apoptotic mechanism, we investigated whether modulation of the PA metabolic pathway impacts the drug-induced apoptotic mechanism. Initially, we determined the expression of the PA biosynthesis enzyme ODC and catabolic enzymes SSAT and PAO in Caco-2 cells following CDK inhibitor treatment for $24 \mathrm{~h}$. As shown in Fig. 3A, while both CDK inhibitors downregulated ODC gene expression, roscovitine and purvalanol upregulated SSAT and PAO protein expression in Caco-2 cells.

Following drug treatments, total PA content of the Caco-2 cells was decreased. The sharp decrease was observed in Spm levels following roscovitine or purvalanol treatments. Although roscovitine led to a significant decrease in Put and Spd levels in Caco-2 cells, purvalanol did not exert any significant effect compared to untreated samples (Fig. 3B). 
A

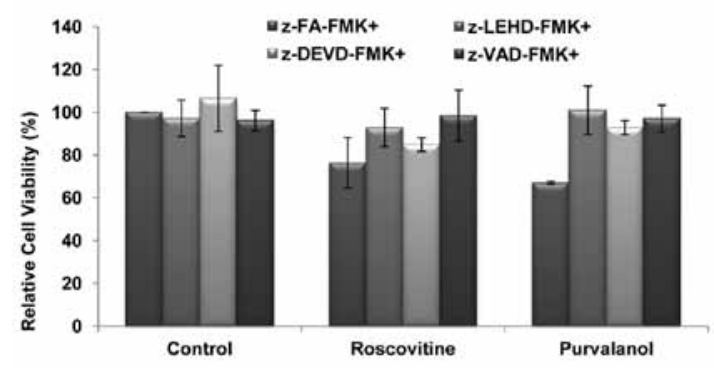

C

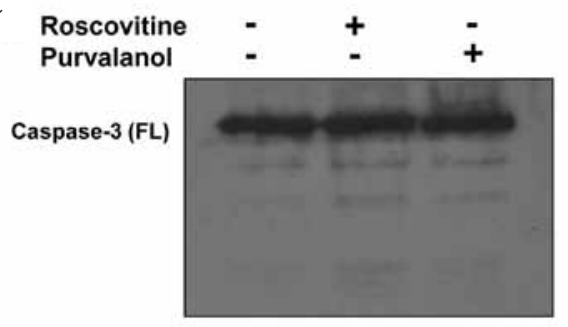

PARP (FL)

PARP (CL)

$\beta$-actin
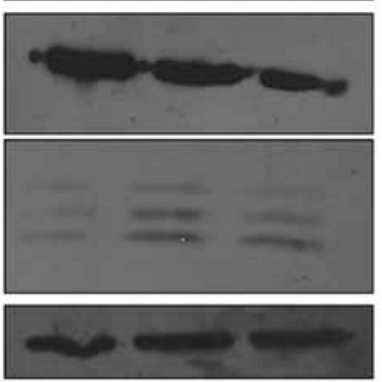

B

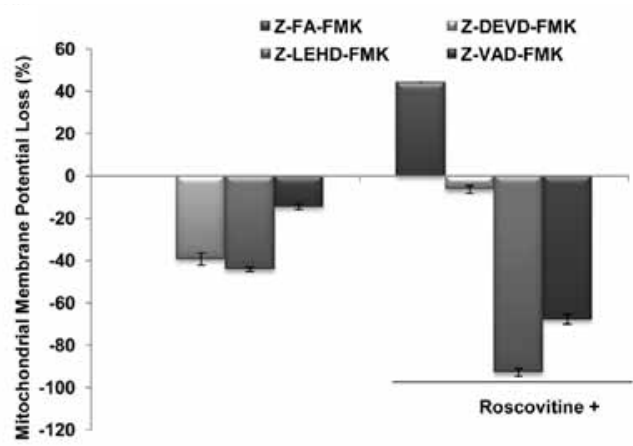

D

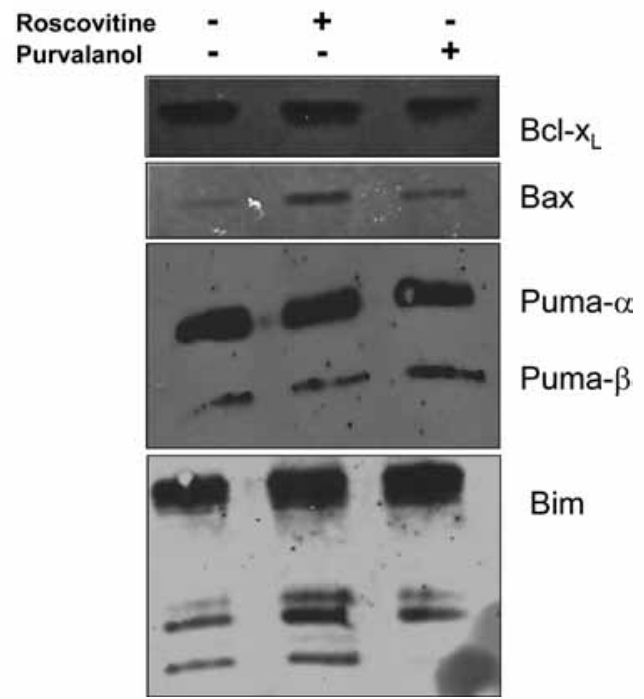

Figure 2. CDK inhibitors induce apoptosis by activating caspases in Caco-2 cells. (A) MTT cell viability assay was performed to evaluate the role of caspases in drug-induced apoptosis. Following $1 \mathrm{~h}$ prior to treatment with caspase-3 (Z-DEVD-FMK), caspase-9 (Z-LEHD-FMK), general caspase (Z-VAD-FMK) and negative caspase (Z-FA-FMK) inhibitors $(2.5 \mu \mathrm{M}$ each), Caco-2 cells were treated with CDK inhibitors roscovitine and purvalanol for $24 \mathrm{~h}$. (B) The disruption of MMP was measured following $\mathrm{DiOC}_{6}$ staining using a fluorometer $(\mathrm{Ex}=485 \mathrm{~nm}$; Em $=538 \mathrm{~nm})$. (C) Activation of caspase-3 and PARP cleavage due to apoptotic induction was determined by immunoblotting. Total protein $(30 \mathrm{mg})$ was loaded into each well. $\beta$-actin was used as loading control. Columns represent the means $\pm \mathrm{SD}$ of five replicates from two different culture conditions. (D) Immunoblotting assay was performed to evaluate the modulation of Bcl-2 family members, such as Bcl- $x_{\mathrm{L}}$, Bax, Puma and Bim, following CDK inhibitor treatment in Caco-2 cells.

A

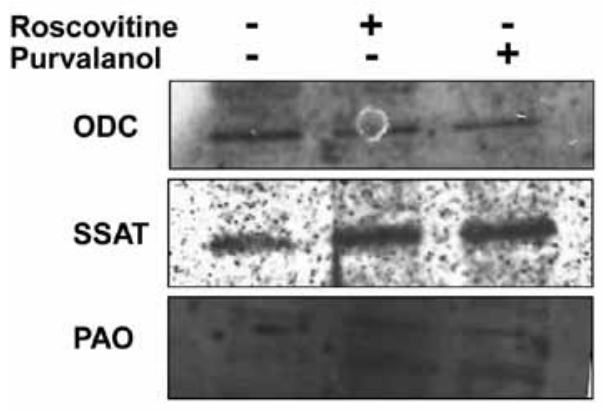

B

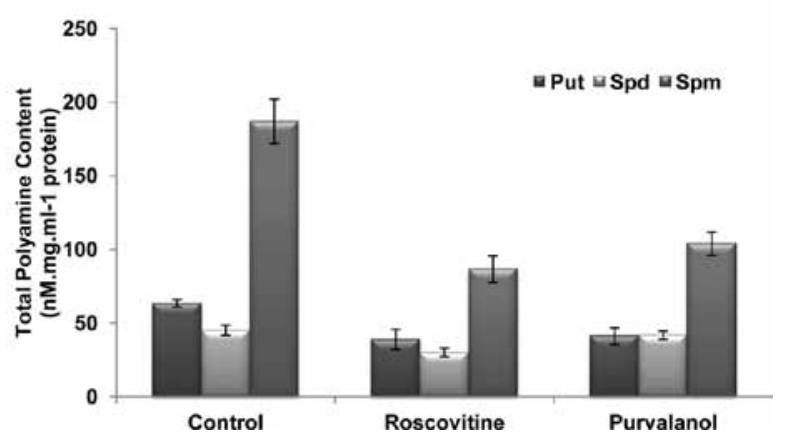

Figure 3. CDK inhibitors alter the expression profiles of polyamine metabolic regulators. (A) Immunoblotting assay was employed to determine the modulation of biosynthetic and catabolic pathway key enzymes ODC, SSAT and PAO, following treatment with CDK inhibitors in Caco-2 cells. (B) Total polyamine content was determined following treatment with CDK inhibitors in Caco-2 cells by HPLC.

SSAT silencing attenuates the apoptotic effect of CDK inhibitors. The efficiency of SSAT siRNAs on SSAT levels was assessed at $48 \mathrm{~h}$ by the treatment of four different siRNA duplexes targeting SAT1 and SAT2 mRNAs, which expressed the SSAT in the human genome. According to SSAT immu- noblotting results, the transfection efficiency of SAT2 (2.6) was the most marked, compared to the other siRNA duplexes. SAT1 mRNA product was downregulated by SAT1.6 siRNA duplex $(2.4 \mu \mathrm{g})$ and SAT2 mRNA was degraded by the treatment of SAT2.7 $(2.4 \mu \mathrm{g})$ following $48 \mathrm{~h}$ of treatment (Fig. 4A). 
A

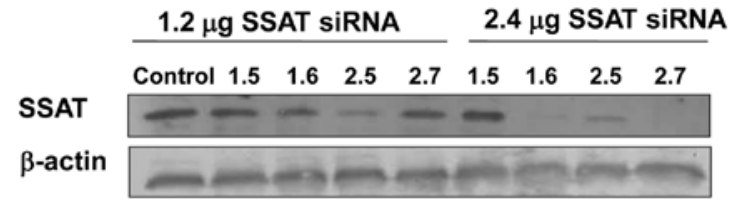

B

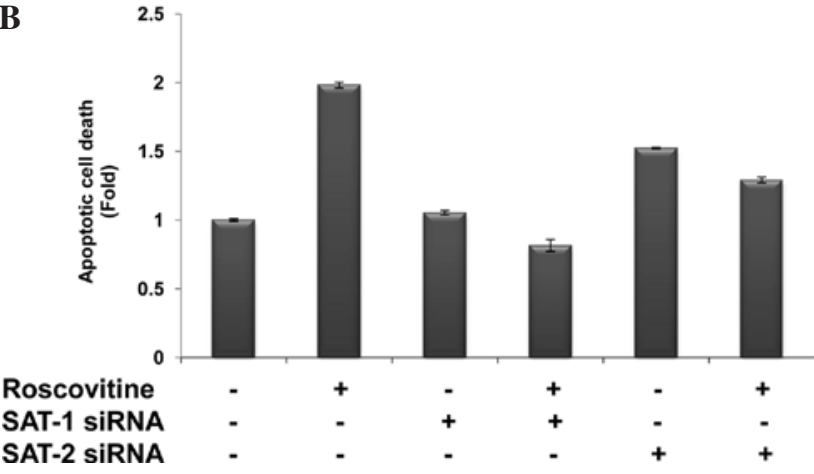

Figure 4. Determination of roscovitine-induced apoptosis in transient silencing of SSAT in Caco-2 cells. (A) Following transfection of Caco-2 cells with SSAT siRNA 1.6, 2.7 and their combination for $48 \mathrm{~h}$, roscovitine treatment was performed for $24 \mathrm{~h}$. SSAT silencing was confirmed by SSAT immunoblotting. Total protein $(30 \mathrm{mg})$ was loaded into each well. $\beta$-actin was used as loading control. (B) CDK inhibitor-induced apoptosis was evaluated in SSAT transiently silenced cells. Columns represent the means \pm SD of two independent experiments with at least four replicates $(" \mathrm{p}<0.05)$.

However, the treatment of SAT1.5 siRNA duplex targeting SAT1 mRNA was not efficient.

The potential role of SSAT in the drug-induced apoptosis mechanism was modelled by the treatment of roscovitine in SSAT-silenced Caco-2 cells (Fig. 4B). Although roscovitine treatment caused a significant 2-fold apoptosis induction compared to untreated samples, roscovitine did not induce apoptosis in SAT1.6 siRNA-transfected cells. According to the immunoblotting results, SAT2.7 siRNA treatment caused a significant downregulation of SSAT expression, and also had a smaller impact on roscovitine-induced apoptotic mechanism in Caco-2 cells.

\section{Discussion}

Genetic alterations in the regulation of the natural PAs Put, Spd and Spm which are essential for cell growth and differentiation may induce cell cycle arrest and apoptosis in various cancer cell lines (36). Recent studies have indicated that chemotherapeutic agents, such as cisplatin, paclitaxel, doxorubicin, 5-FU and oxaliplatin, may induce SSAT expression and decrease total PA content in cells $(37,38)$. It has been shown that 5-FU and oxaliplatin upregulated SSAT expression in a panel of colon cancer cells; H630, HT-29, LoVo, HCT175 and HCT248 colorectal cancer cell lines. Only treatment with oxaliplatin exerted a significant effect on SSAT expression in RKO colorectal cancer cells (39). Moreover, aspirin, a nonsteroidal anti-inflammatory drug, decreases the intracellular PA content by inducing SSAT expression in Caco-2 cells (40). In the present study, we tested the potential role of SSAT, a PA catabolic pathway key enzyme, in the drug-induced apoptotic mechanism in Caco-2 colon cancer cells. To achieve this, cells were treated with CDK inhibitors roscovitine and purvalanol, which are strong apoptotic inducers (Fig. 1A and B). Although both CDK inhibitors lead to apoptotic cell death in various cancer cell types, their targets for apoptotic induction differs. Thus, in order to determine the chemotherapeutic potential of these CDK inhibitors, it is essential to investigate the molecular targets of these CDK inhibitors during apoptotic cell death. According to our previous results, roscovitine induced apoptotic cell death modulated by the PA biosynthetic pathway, and depletion of PAs by a specific inhibitor of ODC increased the roscovitine-induced apoptosis in HCT116 colon carcinoma cells (41). Similar to previous results, we established that roscovitine and purvalanol caspase-dependently induced apoptosis and altered the mitochondrial pathway in Caco-2 cells (Fig. 2). The co-treatment of caspase inhibitors prevented drug-induced cytotoxic effects within $24 \mathrm{~h}$. Consistent with these findings, roscovitine was also shown as a caspase-3 activator in A4573, TC-71 Ewing's sarcoma family of tumour cells, and SK-N-SH neuroblastoma cells and the presence of Ac-DEVD-CHO (a specific caspase-3/-7 inhibitor) prevented roscovitine-induced apoptosis (42). Purvalanol and paclitaxel combined treatment leads to mitochondrial-dependent apoptotic cell death by activating caspase-3 and -9 in HeLa cells (22).

To understand the potential effects of these CDK inhibitors on the PA metabolism, we determined the ODC, SSAT and PAO expression levels following drug treatment for $24 \mathrm{~h}$. As shown in Fig. 3A, while both CDK inhibitors downregulated ODC expression, they upregulated SSAT and PAO gene expression. Moreover, these alterations caused a significant decrease in Put and Spm levels, but no alteration was observed in the Spd content (Fig. 3B). In accordance with these results, PA biosynthesis was inhibited by ODC downregulation and catabolic enzymes were induced by the following treatment of cancer cells with PA analogs, such as PG11047 (37) and classical chemotherapeutic agents (43).

We also tested whether enhanced PA metabolism is critical in the apoptotic decision by transiently transfecting cells with SSAT-targeting siRNA complexes for $48 \mathrm{~h}$. As shown in Fig. 4B, when the induction of SSAT is prevented, Caco-2 cells become more resistant to the apoptotic effects of roscovitine than parental controls. Similar to this finding, the silencing of both SMO and SSAT prevented BENSpm-induced growth inhibitory effect on MDA-MB-231 cells (44).

In summary, these findings show that SSAT is a significant target in the regulation of apoptosis induced by roscovitine. Therefore, the modulation of cellular targets related to PA metabolism may be critical in evaluating the efficiency of drugs in cancer cells. This study also underlines the potential therapeutic efficacy of new generation CDK inhibitors, roscovitine and purvalanol, in colon carcinoma. Although a number of studies have shown the molecular targets of roscovitine, purvalanol has yet to be thoroughly investigated. Therefore, this study is the first to show the therapeutic potential of purvalanol in colon carcinoma cells. Further studies are required to clarify the apoptotic cell death mechanism of purvalanol in colon cancer.

\section{Acknowledgements}

The authors thank Pelin Ozfiliz for the technical assistance during the study. This study was supported by the Istanbul Kultur University Research Fund. 


\section{References}

1. Blagden S and de Bono J: Drugging cell cycle kinases in cancer therapy. Curr Drug Targets 6: 325-335, 2005.

2. Chang KH, de Pablo Y, Lee HP, Lee HG, Smith MA and Shah K: $\mathrm{Cdk} 5$ is a major regulator of $\mathrm{p} 38$ cascade: relevance to neurotoxicity in Alzheimer's disease. J Neurochem 113: 1221-1229, 2010.

3. Schang LM: Advances on cyclin-dependent kinases (CDKs) as novel targets for antiviral drugs. Curr Drug Targets Infect Disord 5: 29-37, 2005.

4. Cicenas $\mathrm{J}$ and Valius M: The CDK inhibitors in cancer research and therapy. J Cancer Res Clin Oncol 137: 1409-1418, 2011.

5. Iseki H, Ko TC, Xue XY, Seapan A, Hellmich MR and Townsend CM Jr: Cyclin-dependent kinase inhibitors block proliferation of human gastric cancer cells. Surgery 122: 187-195, 1997.

6. Meijer L, Borgne A, Mulner O, et al: Biochemical and cellular effects of roscovitine, a potent and selective inhibitor of the cyclindependent kinases cdc2, cdk2 and cdk5. Eur J Biochem 243: 527-536, 1997

7. Schutte B, Nieland L, van Engeland M, Henfling ME, Meijer L and Ramaekers FC: The effect of the cyclin-dependent kinase inhibitor olomoucine on cell cycle kinetics. Exp Cell Res 236 4-15, 1997.

8. Wesierska-Gadek J, Hajek SB, Sarg B, Wandl S, Walzi E and Lindner H: Pleiotropic effects of selective CDK inhibitors on human normal and cancer cells. Biochem Pharmacol 76 $1503-1514,2008$

9. David-Pfeuty T: Potent inhibitors of cyclin-dependent kinase 2 induce nuclear accumulation of wild-type p53 and nucleolar fragmentation in human untransformed and tumor-derived cells. Oncogene 18: 7409-7422, 1999.

10. Appleyard MV, O'Neill MA, Murray KE, et al: Seliciclib (CYC202, R-roscovitine) enhances the antitumor effect of doxorubicin in vivo in a breast cancer xenograft model. Int J Cancer 124: 465-472, 2009.

11. Ortiz-Ferron G, Yerbes R, Eramo A, Lopez-Perez AI, de Maria R and Lopez-Rivas A: Roscovitine sensitizes breast cancer cells to TRAIL-induced apoptosis through a pleiotropic mechanism. Cell Res 18: 664-676, 2008.

12. Wesierska-Gadek J, Kramer MP and Maurer M: Resveratrol modulates roscovitine-mediated cell cycle arrest of human MCF-7 breast cancer cells. Food Chem Toxicol 46: 1327-1333, 2008.

13. Zhang F, Zhang T, Gu ZP, et al: Enhancement of radiosensitivity by roscovitine pretreatment in human non-small cell lung cancer A549 cells. J Radiat Res (Tokyo) 49: 541-548, 2008.

14. Mohapatra S, Chu B, Zhao X, Djeu J, Cheng JQ and Pledger WJ: Apoptosis of metastatic prostate cancer cells by a combination of cyclin-dependent kinase and AKT inhibitors. Int J Biochem Cell Biol 41: 595-602, 2009

15. Maurer M, Komina O and Wesierska-Gadek J: Roscovitine differentially affects asynchronously growing and synchronized human MCF-7 breast cancer cells. Ann NY Acad Sci 1171: 250-256, 2009

16. Zolnierczyk JD, Blonski JZ, Robak T, Kilianska ZM and Wesierska-Gadek J: Roscovitine triggers apoptosis in B-cell chronic lymphocytic leukemia cells with similar efficiency as combinations of conventional purine analogs with cyclophosphamide. Ann NY Acad Sci 1171: 124-131, 2009.

17. Iizuka D, Inanami O, Kashiwakura I and Kuwabara M: Purvalanol A enhances cell killing by inhibiting up-regulation of CDC2 kinase activity in tumor cells irradiated with high doses of $\mathrm{X}$ rays. Radiat Res 167: 563-571, 2007.

18. Cerquetti L, Sampaoli C, Amendola D, et al: Mitotane sensitizes adrenocortical cancer cells to ionizing radiations by involvement of the cyclin B1/CDK complex in G2 arrest and mismatch repair enzymes modulation. Int J Oncol 37: 493-501, 2010.

19. Hikita T, Oneyama C and Okada M: Purvalanol A, a CDK inhibitor, effectively suppresses Src-mediated transformation by inhibiting both CDKs and c-Src. Genes Cells 15: 1051-1062, 2010.

20. Price PM, Yu F, Kaldis P, et al: Dependence of cisplatin-induced cell death in vitro and in vivo on cyclin-dependent kinase 2. J Am Soc Nephrol 17: 2434-2442, 2006.

21. Iizuka D, Ogura A, Kuwabara M and Inanami O: Purvalanol A induces apoptosis and downregulation of antiapoptotic proteins through abrogation of phosphorylation of JAK2/STAT3 and RNA polymerase II. Anticancer Drugs 19: 565-572, 2008.
22. Pennati M, Campbell AJ, Curto M, et al: Potentiation of paclitaxel-induced apoptosis by the novel cyclin-dependent kinase inhibitor NU6140: a possible role for survivin down-regulation. Mol Cancer Ther 4: 1328-1337, 2005.

23. Yenugonda VM, Deb TB, Grindrod SC, et al: Fluorescent cyclindependent kinase inhibitors block the proliferation of human breast cancer cells. Bioorg Med Chem 19: 2714-2725, 2011.

24. Ringer L, Sirajuddin P, Yenugonda VM, et al: VMY-1-103, a dansylated analog of purvalanol B, induces caspase-3-dependent apoptosis in LNCaP prostate cancer cells. Cancer Biol Ther 10: 320-325, 2010.

25. Marton LJ and Pegg AE: Polyamines as targets for therapeutic intervention. Annu Rev Pharmacol Toxicol 35: 55-91, 1995.

26. Marton LJ, Pegg AE and Morris DR: Directions for polyamine research. J Cell Biochem 45: 7-8, 1991.

27. Andersson G and Heby O: Kinetics of cell proliferation and polyamine synthesis during Ehrlich ascites tumor growth. Cancer Res 37: 4361-4366, 1977.

28. Heby O, Andersson G and Gray JW: Interference with S and G2 phase progression by polyamine synthesis inhibitors. Exp Cell Res 111: 461-464, 1978.

29. Bachrach U: Polyamines as chemical markers of malignancy. Ital J Biochem 25: 77-93, 1976

30. Monti MG, Pernecco L, Manfredini R, et al: Inhibition of cell growth by accumulated spermine is associated with a transient alteration of cell cycle progression. Life Sci 58: 2065-2072, 1996.

31. Wang Y and Casero RA Jr: Mammalian polyamine catabolism: a therapeutic target, a pathological problem, or both? J Biochem 139: 17-25, 2006.

32. Casero RA and Pegg AE: Polyamine catabolism and disease. Biochem J 421: 323-338, 2009.

33. Huang Y, Pledgie A, Casero RA Jr and Davidson NE: Molecular mechanisms of polyamine analogs in cancer cells. Anticancer Drugs 16: 229-241, 2005.

34. Chen Y, Weeks RS, Burns MR, Boorman DW, Klein-Szanto A and O'Brien TG: Combination therapy with 2-difluoromethylornithine and a polyamine transport inhibitor against murine squamous cell carcinoma. Int J Cancer 118: 2344-2349, 2006

35. Burns MR, Graminski GF, Weeks RS, Chen Y and O'Brien TG: Lipophilic lysine-spermine conjugates are potent polyamine transport inhibitors for use in combination with a polyamine biosynthesis inhibitor. J Med Chem 52: 1983-1993, 2009.

36. Myhre L, Alm K, Hegardt C, et al: Different cell cycle kinetic effects of N1,N11-diethylnorspermine-induced polyamine depletion in four human breast cancer cell lines. Anticancer Drugs 19: 359-368, 2008.

37. Dredge K, Kink JA, Johnson RM, Bytheway I and Marton LJ: The polyamine analog PG11047 potentiates the antitumor activity of cisplatin and bevacizumab in preclinical models of lung and prostate cancer. Cancer Chemother Pharmacol 65: 191-195, 2009.

38. Hector S, Tummala R, Kisiel ND, et al: Polyamine catabolism in colorectal cancer cells following treatment with oxaliplatin, 5-fluorouracil and N1, N11 diethylnorspermine. Cancer Chemother Pharmacol 62: 517-527, 2008.

39. Allen WL, McLean EG, Boyer J, et al: The role of spermidine/ spermine N1-acetyltransferase in determining response to chemotherapeutic agents in colorectal cancer cells. Mol Cancer Ther 6: 128-137, 2007.

40. Babbar N, Gerner EW and Casero RA Jr: Induction of spermidine/spermine N1-acetyltransferase (SSAT) by aspirin in Caco-2 colon cancer cells. Biochem J 394: 317-324, 2006.

41. Arisan ED, Coker A and Palavan-Unsal N: Polyamine depletion enhances the roscovitine-induced apoptosis through the activation of mitochondria in HCT116 colon carcinoma cells. Amino Acids: Aug 2, 2011 (E-pub ahead of print).

42. Tirado OM, Mateo-Lozano S and Notario V: Roscovitine is an effective inducer of apoptosis of Ewing's sarcoma family tumor cells in vitro and in vivo. Cancer Res 65: 9320-9327, 2005.

43. Varma R, Hector S, Greco WR, et al: Platinum drug effects on the expression of genes in the polyamine pathway: time-course and concentration-effect analysis based on Affymetrix gene expression profiling of A2780 ovarian carcinoma cells. Cancer Chemother Pharmacol 59: 711-723, 2007.

44. Pledgie A, Huang Y, Hacker A, et al: Spermine oxidase SMO(PAOh1), not N1-acetylpolyamine oxidase PAO, is the primary source of cytotoxic $\mathrm{H}_{2} \mathrm{O}_{2}$ in polyamine analogue-treated human breast cancer cell lines. J Biol Chem 280: 39843-39851, 2005. 